\title{
Reliability Risk Evaluation during the Conceptual Design Phase
}

\author{
G. Mamtani, G. Green
}

Systematic evaluations of concept designs involve considering a range of criteria. Interaction with industry supports the view that reliability is a major criterion among those considered in product design. Although there are few methods to predict reliability in the initial phases of design, most of them are only applicable to adaptive designs. In this paper, we introduce the concept of relative reliability risk assessment for original designs, where information availability is less, to calculate reliability. We consider the function structures of the product under consideration and apply the analytic hierarchy process using verbal assessments for relative measurements. The weight assigning technique used is the entropy method. A final value of $R^{3}$ I (Relative Reliability Risk Index) is calculated and the idea of concept functionality graphs is presented. This method is applied on the example of seat suspensions for an off highway vehicle and the results are discussed. The findings help to sort out the concepts that are relatively strong in terms of reliability.

Keywords: reliability, concept design.

Selected paper from the $4^{\text {th }}$ International Conference on Advanced Engineering Design (AED 2004), which was held in Glasgow from 5 to 8 September 2004.

\section{Frame of reference}

Interaction with industry supports the view that reliability is a major criterion [1] under consideration while evaluating concepts in the initial phases of design. Although many multi criteria decision making methods are available to select the final concept (s) from the available candidates [2], reliability like other criterion is normally given some weight and it becomes one of the given criteria during the selection of concepts. Since reliability is a very important criterion in product design, we here propose to obtain the ordinal ranks using subjective inputs on the basis of functionality fulfilment in the initial phases of design. Also, it is amply clear and argued that the data to calculate reliability is not available in the conceptual design phase of original design [3]. However we propose to relatively assess the reliability of concepts and then screen out those that seem to have unacceptable level of rank.

The overview of this paper is as follows. In Section 2, we review reliability and its definition. Section 3 aims to explain the proposed model of comparing concepts with respect to reliability. In the same section, we present an overview of the tools we intend to apply for the calculations. They are the Analytic Hierarchy Process (AHP) [4] and the entropy method [2]. The idea of concept functionality graph is introduced to enable designers to look at the final outcome and to provide ease of decision making. The example problem is introduced in Section 4. It consists of seat suspensions for an off highway vehicle taken from [5]. In Section 5, we apply the method proposed (Section 3) on the example problem (Section 4) and the results are then discussed. We conclude the paper in section 6 with a brief note on our future work.

\section{Reliability: review and definition}

To explore and understand the method proposed, let us first understand the grounds of this research. Reliability has been defined as "The probability that an item will perform a required function, under stated conditions, for a stated period of time." [6]. Normally, while speaking of reliability, we speak in terms of break down of the product. But then this "Breakdown" concept does not fully conform to the above and accepted standard definitions. It is also performance that dictates reliability. For example, if a missile is intended to cover a range of $1000 \mathrm{~km}$ under stated conditions and stated period of time, and if it is unable to reach that range, its reliability is lower.

It is generally argued that conventional reliability calculations in the Conceptual design phase are of limited use [7]. We argue that there are various types of designs that industries undertake and the definition of Conceptual design differs from company to company. For example, a company may wish to utilize the available components in the market for a new product. The product is definitely new but the conceptual design phase of such a product would entail selection of available components to make an "ideal" fit that the industry wishes to go ahead with. Predicting and calculating reliability in such cases is possible using the techniques available. Also, in case of original designs, a Relative measure can be obtained which is explained in the section that follows.

Cooper \& Thompson [8] have listed all the valuable reliability prediction tools in their paper. Qualitative methods have been suggested in the conceptual design phase and quantitative methods in the latter phases of design. Still, most of the techniques applied are meant for adaptive designs [3] or "proprietary products" and not for original designs.

As regards original design [3], absolute reliability calculations in the conceptual design phase are not possible but a relative reliability indicator may be calculated in order to rate the generated design options and get the ordinal rankings for them. We propose a method to utilize functionalities for calculating the index, what we call the Relative Reliability Risk Index (RRRI or $\mathrm{R}^{3} \mathrm{I}$ ). The argument that functionality has less to do with reliability seems invalid here because, as we stated earlier, performance is a measure of reliability and the functionality indication refers to the performance of the product considered during the conceptual design phase. Henceforth, 
we follow a relative approach in calculating $\mathrm{R}^{3} \mathrm{I}$ using the analytic hierarchy process (AHP). The method is proposed in the next section.

\section{Functionality as an indicator of risk and proposed method}

To calculate the Relative Reliability Risk Index $\left(\mathrm{R}^{3} \mathrm{I}\right)$, we propose a four step methodology (Fig. 1). To begin with, we consider the established function structure of the product. We deal with this in more detail in the next sub-section. After consideration of function structure, the Analytic Hierarchy Process (AHP) [4] is applied so as to relatively rate the main functions of the function structure. We consider the main functions (Section 3.1), functions that are fundamental to the system [3] and compare them with respect to the alternatives. After the comparisons have been made, we obtain the priorities. Application of AHP is done using the commercially available Decision Support Software by Expert Choice. The software is interactive with the required number crunching and provides a measure of Inconsistencies during the comparison. This Inconsistency gives a good measure of the relative ratings, and provides a check whether the comparisons should be performed again. Using these priorities, we draw Concept Functionality Graphs (CFGs).

CFG indicates the relative measure of functionality fulfilment with respect to each of the available concepts. The example problem (Section 4) and the application of this methodology on this example shall clarify the steps of this methodology in due course. Step four includes assigning weights to the functions. We do this using the entropy method [2]. This method has been adopted because it does not require the designer to indicate the weight. Instead, weights are calculated using the information obtained from the decision matrix. Additionally, this helps to rule out any chance of prejudice or manipulation to assign weights by the decision- -maker. Even if the weights have already been assigned by the decision-maker, they can be combined with the weights obtained using this method (Section 3.3). Now, this decision matrix is arrived at in step 2 using AHP. The application of AHP leads to the normalized priorities, which are used to extract information for input to the entropy method in step 4.

\subsection{Function structures as a means of modelling concepts}

Establishing Function structures in the Conceptual phase of design helps to pursue design in a systematic manner. There have been many approaches towards developing function models. For brevity, we do not discuss all of them here but follow the approach proposed by Pahl and Beitz [3]. In the initial stages of design, the technical systems are represented using function structures before their solution principles have been proposed. Initially a "Black box" approach towards the system is established, representing the overall system goal with the inputs and outputs. The inputs and outputs are in the form of energy, matter and signals. Then subfunctions are added to this system and each of them is usually represented as a verb-noun pair. The detail of the structure depends on the level of abstraction one wants to achieve. There are two types of functions, main functions and auxiliary functions. Main functions are the those directly help achieve the overall goal, and Auxiliary functions indirectly help in achieving the overall function. To understand this better, let us take an example of a common 3-axes horizontal Lathe machine. The function structure of such a Lathe is shown in Fig. 2 and Fig. 3 at different levels of abstraction.

Initially the overall function is laid down in which the main task of the Lathe is considered i.e. Machining Work piece (W/P) (As shown in Fig. 2). To understand this, refer to the symbols for the conversion of matter, energy and signals as shown below Fig. 3. When considered at a detailed level of abstraction, the structure as shown in Fig. 3 is arrived at.

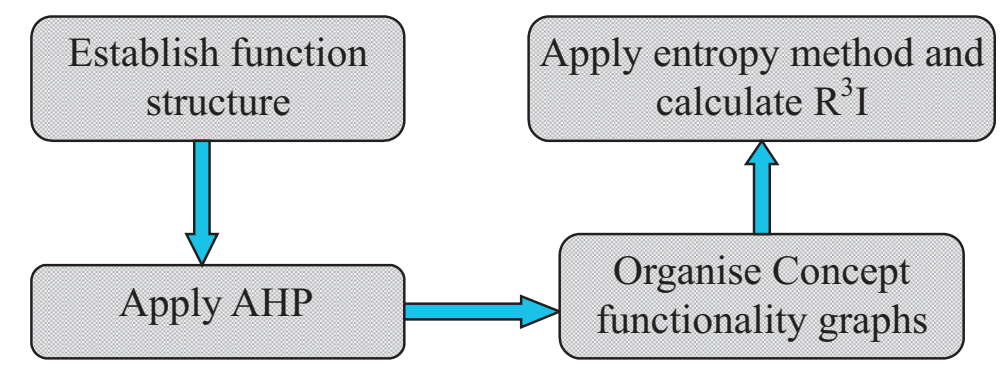

Fig. 1: Steps to calculate $\mathrm{R}^{3} \mathrm{I}$

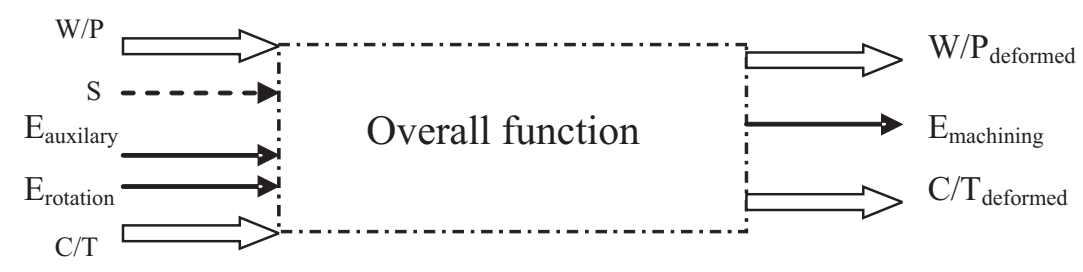

Fig. 2: "Black Box" approach 


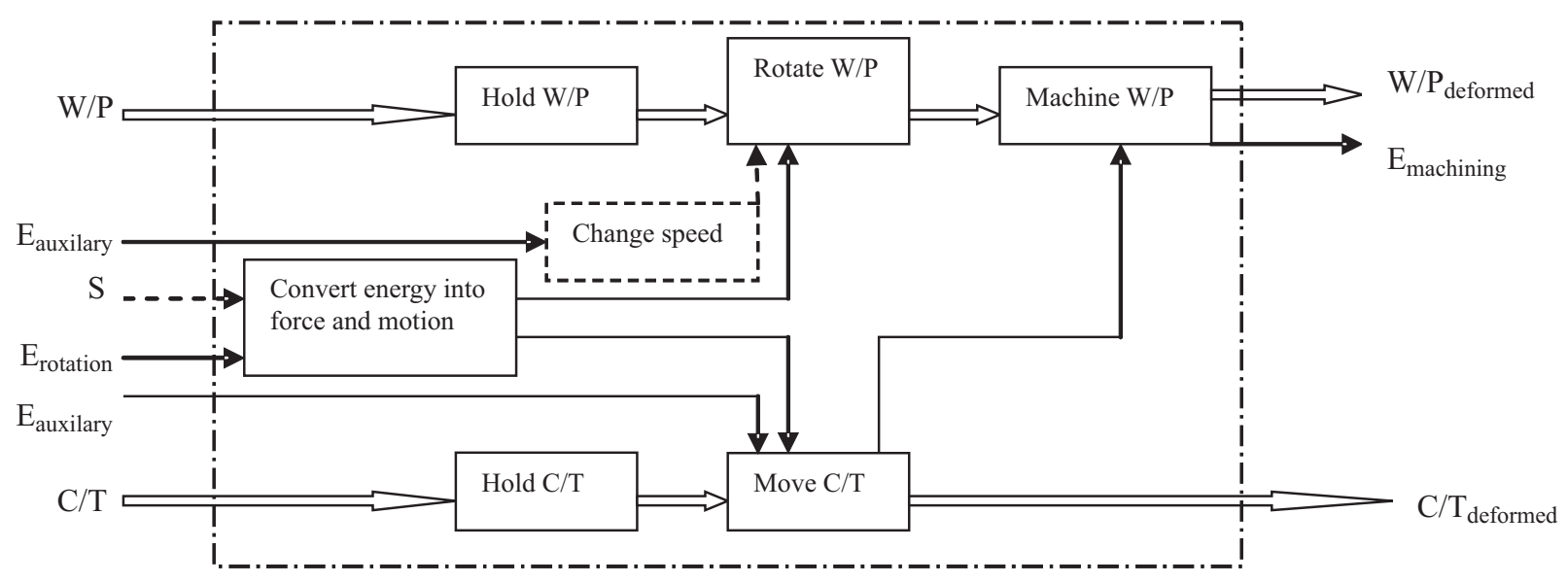

Where,

Energy

Signals

$\mathrm{C} / \mathrm{T}$

Main function

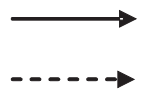

Cutting tool

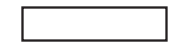

Materials

$\mathrm{W} / \mathrm{P}$

System boundary

Auxiliary function

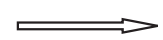

Work piece

-...-........

ín

Fig. 3: Function structure of a Lathe

\subsection{Analytic hierarchy process}

The analytic hierarchy process, developed by Saaty, is one of the available mutli attribute decision making tools. The strength of this tool lies in utilising insight based soft information from the decision makers in the form of relative values. A hierarchy is developed in which the main objective forms the highest level. The next lower level is occupied by the criteria, and so on. The bottom most hierarchy is occupied by the alternatives available. One such hierarchy is shown in Fig. 4. Once the hierarchy has been established, comparison matrices are formulated and comparisons of lower level criteria are made with respect to the property at the upper level. Much literature is available on AHP that deals with the mathematics of the method, one of them being [9].

The example problem we undertake to illustrate the AHP method is that of selecting a temperature sensor. A University Thermodynamics Laboratory wants to purchase a temperature sensor for temperature measurements. The al- ternatives available in the market are thermistors, platinum resistance thermometers and thermocouples. We would like to mention here that this is a hypothetical situation where we limit out alternatives to three only for ease of explanation. The criteria on which the selection depends are accuracy, temperature range measured, price and reliability. The hierarchy is shown in Fig. 4. If we apply the top-down approach here, we would first compare all the criteria, i.e. accuracy, temperature range, price and reliability using a pairwise comparison matrix with respect to the objective i.e. selecting a temperature sensor. Such a pairwise comparison matrix is shown in Table 1. Next, we compare all the three alternatives with respect to each property at the level above it. There would be four comparison matrices for these comparisons that are shown as Table 2, 3, 4 and 5. Comparisons are made using a scale that involves integers from 1 to 9 and their reciprocals to represent relative importance. If a numeric scale cannot be used, verbal assessment is then preferred. We shall be using verbal assessment for calculating $\mathrm{R}^{3} \mathrm{I}$.

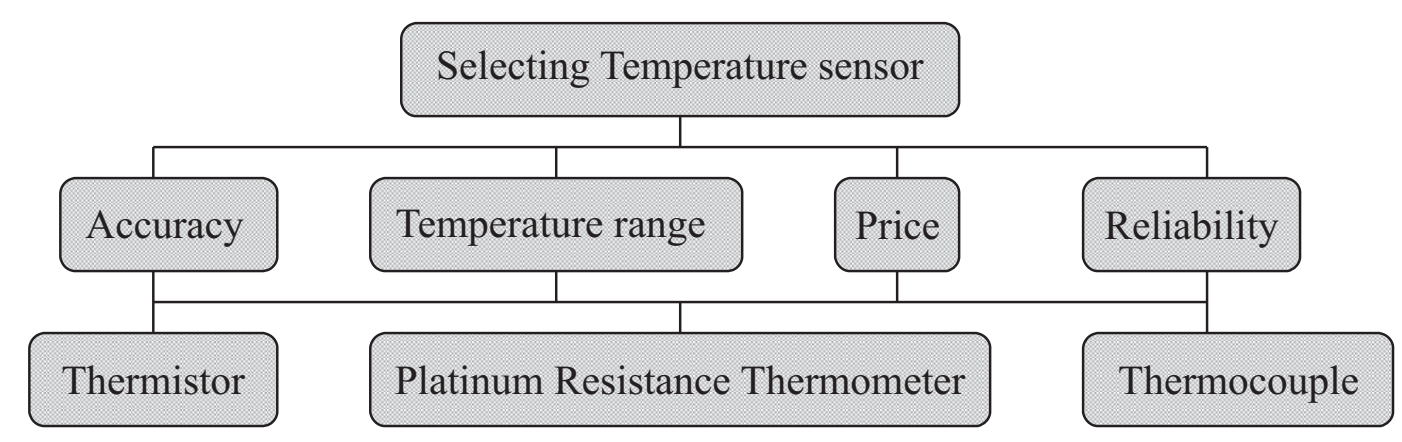

Fig. 4: Hierarchy for a temperature sensor selection problem 
Table 1: Comparison matrix for criteria

\begin{tabular}{|l|c|c|c|c|c|}
\hline Sensor & A & TR & P & R & Priorities \\
\hline A & 1 & 3 & $1 / 3$ & 2 & .257 \\
\hline TR & $1 / 3$ & 1 & $1 / 3$ & 2 & .147 \\
\hline P & 3 & 3 & 1 & 3 & .483 \\
\hline R & $1 / 2$ & 3 & $1 / 3$ & 1 & .113 \\
\hline
\end{tabular}

Incon: 0.08

Table 2: Comparison matrix wrt Accuracy

\begin{tabular}{|l|c|c|c|c|}
\hline $\mathrm{A}$ & PRT & $\mathrm{T}$ & $\mathrm{TC}$ & Priorities \\
\hline PRT & 1 & 5 & 5 & .709 \\
\hline $\mathrm{T}$ & $1 / 5$ & 1 & 2 & .179 \\
\hline $\mathrm{TC}$ & $1 / 5$ & $1 / 2$ & 1 & .113 \\
\hline
\end{tabular}

Incon: 0.05

Table 3: Comparison matrix wrt Temp range

\begin{tabular}{|l|c|c|c|c|}
\hline TR & PRT & T & TC & Priorities \\
\hline PRT & 1 & 5 & 6 & .726 \\
\hline $\mathrm{T}$ & $1 / 5$ & 1 & 2 & .172 \\
\hline TC & $1 / 6$ & $1 / 2$ & 1 & .102 \\
\hline
\end{tabular}

Incon: 0.03

Table 4: Comparison matrix wrt Reliability

\begin{tabular}{|l|c|c|c|c|}
\hline $\mathrm{R}$ & PRT & $\mathrm{T}$ & $\mathrm{TC}$ & Priorities \\
\hline PRT & 1 & 3 & 5 & .163 \\
\hline $\mathrm{T}$ & $1 / 3$ & 1 & 3 & .540 \\
\hline $\mathrm{TC}$ & $1 / 5$ & $1 / 3$ & 1 & .297 \\
\hline
\end{tabular}

Incon: 0.01

Table 5: Comparison matrix wrt Price

\begin{tabular}{|l|c|c|c|c|}
\hline $\mathrm{P}$ & PRT & $\mathrm{T}$ & $\mathrm{TC}$ & Priorities \\
\hline PRT & 1 & $1 / 3$ & $1 / 2$ & .637 \\
\hline $\mathrm{T}$ & 3 & 1 & 2 & .258 \\
\hline $\mathrm{TC}$ & 2 & $1 / 2$ & 1 & .105 \\
\hline
\end{tabular}

Incon: 0.04

Here, $\mathrm{A}=$ accuracy, $\mathrm{TR}=$ temperature range measured, $\mathrm{R}=$ reliability, $\mathrm{P}=$ price, $\mathrm{PRT}=$ platinum resistance thermometer, $\mathrm{T}=$ thermistor and $\mathrm{TC}=$ thermocouple.

The priorities calculated are shown in the comparison matrices. These matrices are used to calculate the final priorities for the available alternatives. With each matrix, there is associated a Consistency Ratio (CR) which gives the measure of consistency in the comparisons made. We use the expert choice software for calculating CR. Usually, CR should be under $10 \%$ for the results to be acceptable; else the comparison should be undertaken again.

In the method we have proposed to calculate $\mathrm{R}^{3} \mathrm{I}$, we shall calculate the priorities of the alternatives with respect to criteria, but we do not compare the criteria with respect to the objective. This is because the criteria that are available with us are functions (Main functions) from the function structures. It would be inadvisable to compare the functions that are basic or fundamental to the system using the pairwaise comparison matrix, because all the main functions may seem to be equally important to the designer. Instead, we use the Entropy method [2] to calculate the weights of the functions with us. Sub section 3.3 explains the entropy method.

\subsection{Entropy method to calculate weights}

The entropy method [2] is an MADM method to calculate the weights of the attributes that have been considered during the decision-making process. It utilizes the information content of the decision matrix to calculate the weights of the attributes. This method has been adopted as a part of calculating $\mathrm{R}^{3} \mathrm{I}$ because it may be inappropriate for a designer to compare functions relatively from the function structure. The information contents of the normalized values of the attributes can be measured using entropy values. The entropy $V_{j}$ of the set of normalized outcomes of attribute $j$ is given by

$V_{j}=-\beta \sum_{i=1}^{n} l_{i j} * \ln l_{i j}$ for all $j,(j=1$ to $k$ represents

attribute and $i=1$ to $n$ represents alternative)

where $\beta$ is constant which defined as $\beta=1 / \ln (n)$ and $l_{i j}$ is a normalized element of the decision matrix. If there are no preferences available, the weights are calculated using the equation

$$
w_{j}=\frac{e_{j}}{\sum_{j=1}^{k} e_{j}} \text { and } e_{j}=1-V_{j} .
$$

If the decision maker has the weights available beforehand $w_{e}$, it can be combined with the weights calculated above, resulting in new weights that are $w_{\text {new }}$.

$$
W_{\text {new }}=\frac{w_{e} * w_{j}}{\sum_{j=1}^{k} w_{e} * w_{j}} .
$$

\subsection{Concept functionality graphs}

Concept functionality graphs depict the strengths and weaknesses of the concepts generated in the conceptual design phase. They are the graphs between the functional priorities obtained from AHP and the concepts. Ulrich \& Eppinger [10] have proposed a five-step method for generating solution concepts using Function diagrams. This strategi c approach towards generating concepts helps identify the strengths and weaknesses of all concepts functionwise. Unfortunately, systematic methods are not always used in industry [11]. Also, a large number of concepts generated produce a complex situation to recognise the strengths and weaknesses as regards each function in the concepts. Henceforth concept 
functionality graphs are thought of as a means to represent the strengths and weaknesses of concepts after the comparison using AHP has been performed.

\section{Example under consideration: seat suspensions}

The example we use here to illustrate the application of this methodology is the seat suspension mechanism for off-highway vehicles. It has been taken from [5]. Hurst had considered this example to illustrate the effectiveness of using spreadsheets for concept selection. The method applied is similar to the Weighting \& Rating method and the ratings provided to the concepts with respect to criteria are in terms of satisfaction of criteria. All the six concepts are shown in Fig. 5.

\section{Application of the proposed method on example and results}

\subsection{Establishing function structures}

The function structure established for Seat suspensions is shown in Fig. 6. Essentially, 3 main functions are considered in the structure. They are Hold seat, Dampening Vibrations and Adjusting Seat height. The flow of matter, energy and signals are shown.

\subsection{Applying AHP for comparing concepts with respect to the main functions}

AHP is applied to the functions considered here and the comparison matrices are shown in Tables 6, 7 and 8. In Tables 6, 7 and 8, A, B etc refer to Concept A, B etc. The inconsisten-

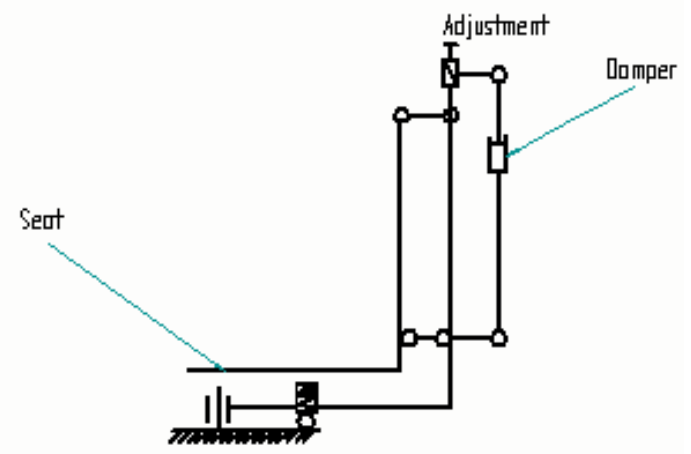

Concept A

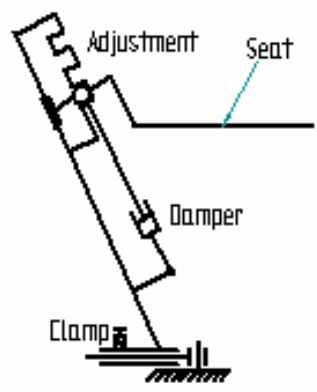

Concept C

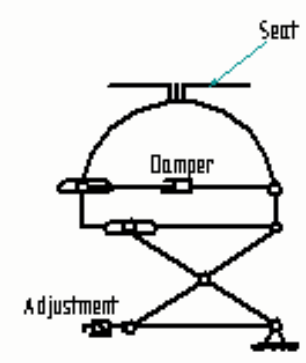

Concept E

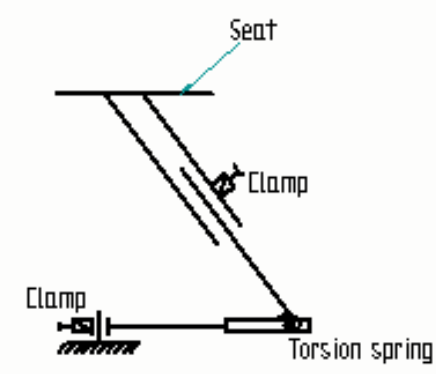

Concept B

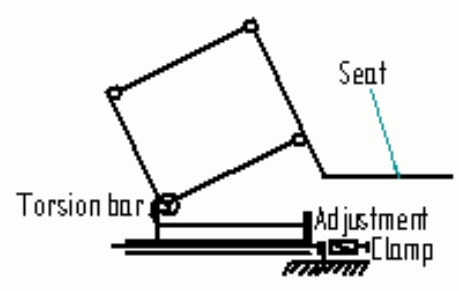

Concept D

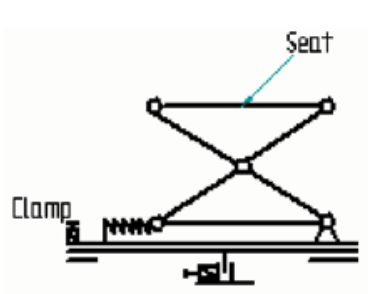

Concept F

Fig. 5: Concepts for seat suspensions for off-highway vehicles (After Hurst) 


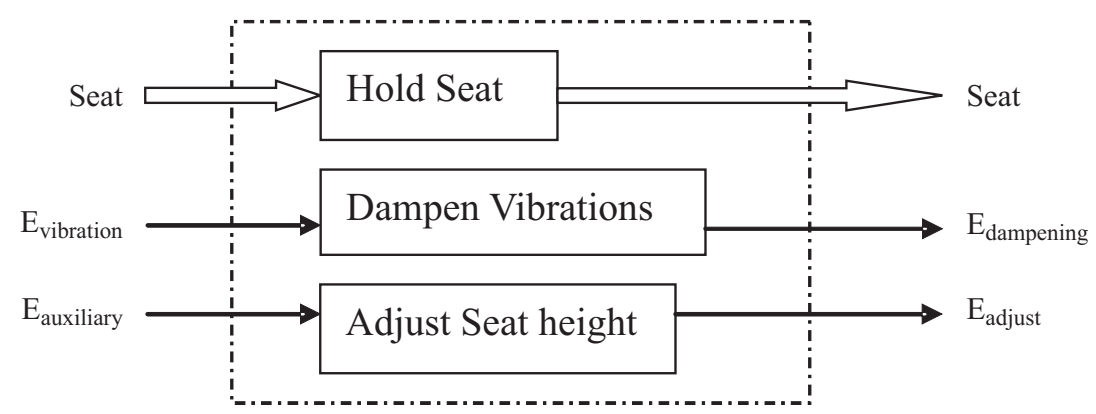

Fig. 6: Function structure of seat suspension mechanism

Table 6: Comparison matrix wrt to Hold Seat

\begin{tabular}{|c|c|c|c|c|c|c|c|}
\hline Hold seat & A & B & C & D & E & F & Priorities \\
\hline A & 1 & 5 & 3 & 3 & 2 & $1 / 3$ & 0.233 \\
\hline B & $1 / 5$ & 1 & $1 / 3$ & 2 & $1 / 3$ & $1 / 4$ & 0.061 \\
\hline C & $1 / 3$ & 3 & 1 & 3 & $1 / 3$ & $1 / 4$ & 0.103 \\
\hline D & $1 / 3$ & $1 / 2$ & $1 / 3$ & 1 & $1 / 5$ & $1 / 5$ & 0.047 \\
\hline E & $1 / 2$ & 3 & 3 & 5 & 1 & $1 / 2$ & 0.190 \\
\hline F & 3 & 4 & 4 & 5 & 2 & 1 & 0.365 \\
\hline
\end{tabular}

Incon.: 0.06

Table 7: Comparison matrix wrt to Dampen Vibrations

\begin{tabular}{|c|c|c|c|c|c|c|c|}
\hline $\begin{array}{c}\text { Dampen } \\
\text { vibrations }\end{array}$ & A & B & C & D & E & F & Priorities \\
\hline A & 1 & 5 & 3 & 5 & $1 / 2$ & 3 & 0.0271 \\
\hline B & $1 / 5$ & 1 & $1 / 3$ & 1 & $1 / 5$ & $1 / 2$ & 0.053 \\
\hline C & $1 / 3$ & 3 & 1 & 3 & $1 / 4$ & 3 & 0.145 \\
\hline D & $1 / 5$ & 1 & $1 / 3$ & 1 & $1 / 5$ & $1 / 2$ & 0.053 \\
\hline E & 2 & 5 & 4 & 5 & 1 & 5 & 0.396 \\
\hline F & $1 / 3$ & 2 & $1 / 3$ & 2 & $1 / 5$ & 1 & 0.082 \\
\hline
\end{tabular}

Incon.: 0.03

Table 8: Comparison matrix wrt to Adjust Seat height

\begin{tabular}{|c|c|c|c|c|c|c|c|}
\hline $\begin{array}{c}\text { Adjust Seat } \\
\text { Height }\end{array}$ & A & B & C & D & E & F & Priorities \\
\hline A & 1 & $1 / 3$ & $1 / 2$ & 1 & $1 / 2$ & $1 / 2$ & 0.082 \\
\hline B & 3 & 1 & 3 & 5 & 2 & 3 & 0.352 \\
\hline C & 2 & $1 / 3$ & 1 & 3 & $1 / 2$ & 2 & 0.157 \\
\hline D & 1 & $1 / 5$ & $1 / 3$ & 1 & $1 / 3$ & $1 / 3$ & 0.061 \\
\hline E & 2 & $1 / 2$ & 2 & 3 & 1 & 3 & 0.229 \\
\hline F & 2 & $1 / 3$ & $1 / 2$ & 3 & $1 / 3$ & 1 & 0.119 \\
\hline
\end{tabular}

Incon.: 0.04

(C) Czech Technical University Publishing House

$\underline{\text { http://ctn.cvut.cz/ap/ }}$ cies are also laid down with each matrix considered. The inconsistencies are all less than 0.1 and are acceptable. After the application of AHP, a priority matrix is obtained (Table 9). This will be treated as our decision matrix.

Table 9: Priority matrix for seat suspension concepts

\begin{tabular}{|l|c|c|c|c|c|c|}
\hline \multirow{2}{*}{} & \multicolumn{6}{|c|}{ Concept } \\
\cline { 2 - 7 } & A & B & C & D & E & F \\
\hline Hold seat & 0.233 & 0.061 & 0.103 & 0.047 & 0.19 & 0.365 \\
\hline $\begin{array}{l}\text { Dampen } \\
\text { vibrations }\end{array}$ & 0.271 & 0.053 & 0.145 & 0.053 & 0.396 & 0.082 \\
\hline Adjust seat height & 0.082 & 0.352 & 0.157 & 0.061 & 0.229 & 0.119 \\
\hline
\end{tabular}

\subsection{Concept functionality graphs for the example considered}

The CFG for this example is shown in Fig. 7. The integers $1-6$ on the X-axis in Fig. 7 represent Concept A - Concept F respectively. The figure is meant to depict a clear picture of the strengths and weaknesses of different concepts with respect to the functions considered.

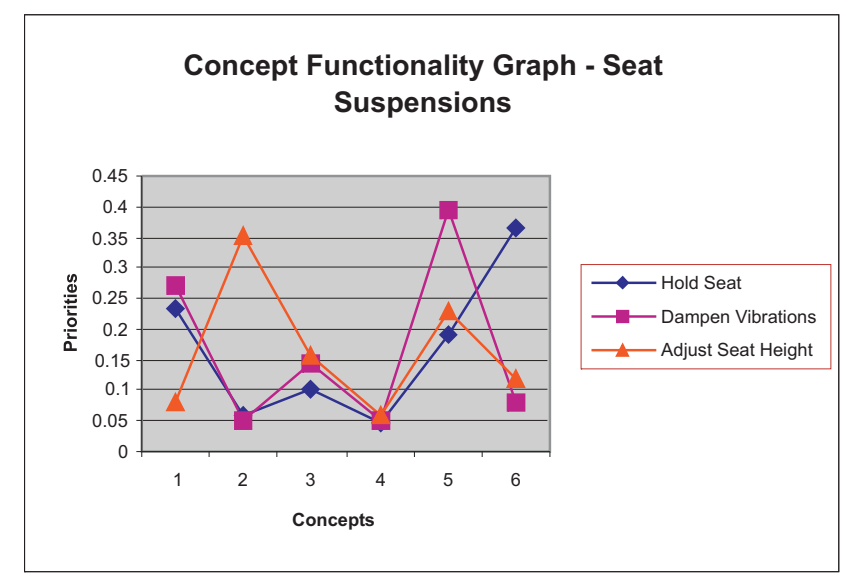

Fig. 7: CFG for the seat suspension example

\subsection{Application of the entropy method to calculate weights}

The weights for the three functions considered have been calculated using the information from the matrix and the entropy method (explained in Section 3.3) is utilized to calculate 
the same. The weights obtained after the application of the method are shown in Table 10. Normalisation of the decision matrix is not required since the sum of priorities for any attribute $j$ is 1 in Table 9 .

Having calculated the weights and priorities, we obtain $\mathrm{R}^{3} \mathrm{I}$ (Table 11) using Eq. (4).

$$
\mathrm{R}^{3} \mathrm{I}_{i}=\sum_{j=1}^{k} l_{i j} * w_{j} \text { for all } i \text {. }
$$

We can see from Table 11 that Concept $\mathrm{E}$ has the best $\mathrm{R}^{3} \mathrm{I}$ among all the available concepts. Also the concepts that may be screened out are those that have low $\mathrm{R}^{3} \mathrm{I}$ value, which are $\mathrm{B}$ and D. The ordinal ranks are also shown in Table 11.

Table 10: Weights obtained after application of the entropy method

\begin{tabular}{|l|c|}
\hline Functions & Weight $\left(w_{j}\right)$ \\
\hline Hold seat & 0.33 \\
\hline Dampen vibrations & 0.413 \\
\hline Adjust seat height & 0.252 \\
\hline
\end{tabular}

Table 11: $\mathrm{R}^{3} \mathrm{I}$ and ranks for concepts - seat suspensions

\begin{tabular}{|l|c|c|c|c|c|c|}
\hline & $\mathrm{A}$ & $\mathrm{B}$ & $\mathrm{C}$ & $\mathrm{D}$ & $\mathrm{E}$ & $\mathrm{F}$ \\
\hline $\mathrm{R}^{3} \mathrm{I}$ & 0.209 & 0.13 & 0.133 & 0.052 & 0.283 & 0.184 \\
\hline Rank & 2 & 5 & 4 & 6 & 1 & 3 \\
\hline
\end{tabular}

\section{Conclusion}

In this paper, we reviewed reliability and proposed a method for calculating a relative index to compare concepts in the initial phases of design. The method helps to obtain ordinal rankings of the available concepts and is applied on the example of seat suspensions for off highway vehicles. The methodology involves application of the analytic hierarchy process to relatively compare concepts and the entropy method for obtaining the weights of the functions considered. The idea of concept functionality graphs is introduced and the results of application on the example are discussed. Future work includes validation of this methodology using other examples from student projects and from industry.

\section{References}

[1] Mamtani, G., Green, G.: "Evolution of a Computer Evaluation Tool in Context with Scottish Industries", Proceedings of Design 2004 conference, Dubrovnik, 2004.

[2] Sen, P., Yang, J.: Multiple Criteria Decision Support in Engineering Design. London, 1998.

[3] Pahl, G., Beitz, W.: Engineering Design - A Systematic Approach. Ken Wallace, London, 1996.

[4] Saaty, Thomas L.: Decision Making for Leaders. Pittsburgh, 2001.

[5] Hurst, K.: "Spreadsheet Analysis Applied to the Concept Selection Phase of Engineering Design." Proceedings of International Conference on Engineering Design, Dubrovnik, 1990.

[6] Smith, D.: Reliability and Maintainability in Perspective. London, 1988.

[7] Smith, J., Clarkson, P. J.: "Improving Reliability during Conceptual Design.” Proceedings of International Conference on Engineering Design, Glasgow, 2001.

[8] Cooper, G., Thompson, G.: "Concept Design and Reliability." Advanced Engineering Design, Glasgow, 2001.

[9] Saaty, Thomas L.: The Fundamentals of Decision Making and Priority Theory with the Analytic Hierarchy Process. Pittsburgh, 2000.

[10] Ulrich, K., Eppinger, S.: Product Design and Development. Boston, 2000.

[11] Taylor, Ben: "Enhancement of Design Evaluation during Concept Development.” Proceedings of International Conference on Engineering Design, The Hague Netherlands, 1993.

Girish Mamtani

e-mail: g.mamtani@mech.gla.ac.uk

Dr. Graham Green

phone: +44(0) 1413304071

e-mail: g.green@mech.gla.ac.uk

Mechanical Engineering Department

James Watt Building

University of Glasgow

Glasgow, G12 8QQ

Scotland, UK 